\title{
AutoReg: uma ferramenta de apoio à autorregulação da aprendizagem
}

\author{
José Carlos M. da Silva Júnior ${ }^{1}$, Rozelma S. de França ${ }^{1}$, Patrícia C. de A. R. Tedesco ${ }^{1}$ \\ ${ }^{1}$ Centro de Informática - Universidade Federal de Pernambuco (CIn - UFPE) \\ Recife - PE - Brasil \\ $\{j \mathrm{cmsj}$, rsf2, pcart\}@cin.ufpe.br
}

\begin{abstract}
Nowadays it is necessary to develop, during the school education, skills to enable students to manage their own knowledge. In this context, training in selfregulatory skills can contribute with this goal. Thus, this work presents Autoreg, a tool developed to support the self-regulation of student learning in its formation process. In addition to their requirements and architecture, is presented a usage scenario with Autorreg, aiming to show some benefits provided to their use.
\end{abstract}

Resumo. Atualmente é necessário desenvolver, durante a formação escolar, habilidades que possibilitem aos estudantes gerenciar o próprio conhecimento. Neste contexto, o treinamento em habilidades autorregulatórias pode contribuir com este objetivo. Desse modo, este trabalho apresenta a AutoReg, uma ferramenta desenvolvida para apoiar a autorregulação da aprendizagem dos estudantes em seu processo de formação. Além de seus requisitos e arquitetura, é apresentado um cenário de uso com a AutoReg, visando mostrar alguns beneficios providos com a sua utilização.

\section{Introdução}

Cada vez mais é necessário criar cenários que possibilitem a formação de estudantes ativos em sua própria aprendizagem contribuindo, assim, para o desenvolvimento de habilidades requeridas desses sujeitos no século XXI. Neste contexto, autorregulação da aprendizagem é definida como uma habilidade a ser desenvolvida no ambiente escolar na qual, segundo Zimmerman (1990), os estudantes planejam, monitoram e avaliam o próprio conhecimento durante o processo de aprendizagem. Evidências dos efeitos da autorregulação no desenvolvimento dos aprendizes podem ser conferidas em estudos como os de Kumar et al. (2005) e Alhazbi et al. (2010).

Considerando o exposto e o crescente uso da tecnologia na educação, neste trabalho é apresentada a AutoReg, uma ferramenta que tem como objetivo dar suporte ao desenvolvimento da autorregulação da aprendizagem dos estudantes. A ferramenta auxilia os alunos no planejamento de atividades, definição de metas de estudo e monitoramento do conhecimento. Além disso, possui um mecanismo que possibilita a troca de mensagens entre estudantes e professor. Ao professor, cabe orientar, sugerir melhorias nas atividades e metas propostas pelos alunos. A estes, além de planejar, monitorar e avaliar o próprio aprendizado, cabe contribuir com a formação de outros estudantes que tenham compartilhado com a turma o seu planejamento de estudo.

Assim, além desta seção introdutória, este artigo traz na seção 2 estudos que descrevem experiências e ferramentas de apoio à autorregulação; na seção 3 é descrita a arquitetura da ferramenta desenvolvida e seus requisitos; a seção 4 mostra o funcionamento da AutoReg por 
meio de um cenário de uso e na seção 5 são feitas algumas considerações finais a cerca de todo o trabalho.

\section{Trabalhos relacionados}

Durante a pesquisa realizada foram encontrados diversos trabalhos sobre autorregulação da aprendizagem. Para a análise e comparação dos estudos correlatos com a ferramenta aqui apresentada, o foco da descrição a seguir será sobre os requisitos de gerenciamento de atividades pelos usuários e comunicação entre esses sujeitos suportados pelas ferramentas.

Lima et al. (2013) propõem uma ferramenta que incorpora mecanismos para a autorregulação na qual os estudantes realizam avaliações e acompanham o nível de conhecimento adquirido, provendo informações para que eles conscientizem-se sobre o processo de aprendizagem. Entretanto, essa ferramenta não permite a interação entre estudantes, aspecto importante na medida em que os pares podem ajudar determinado estudante no gerenciamento de sua aprendizagem.

Já no trabalho de Sirotheau et al. (2011), foi integrado a um ambiente virtual de aprendizagem funcionalidades que permitem o feedback entre os estudantes como forma de estimular o desenvolvimento da habilidade de autoavaliação. Tais funcionalidades permitem que os estudantes, além de enviar soluções para os exercícios propostos, também avaliem propostas de outros estudantes. Por outro lado, a ferramenta em questão, apesar de permitir a interação entre aprendizes não oferece outros recursos como gerenciamento do curso pelo professor e gerenciamento das atividades pelos estudantes.

\section{A ferramenta AutoReg}

Esta seção apresenta a ferramenta Autorreg que incorpora mecanismos de apoio à autorregulação da aprendizagem.

\subsection{Requisitos}

Na ferramenta desenvolvida, há a Agenda da Disciplina que permite ao professor criar a agenda de uma disciplina. Para cada atividade da agenda, o professor deve informar conteúdo, data de início e término da atividade, sugestão de materiais, sugestão de estratégias de aprendizagem. Após criar a agenda da disciplina, a mesma poderá ser acessada pelos estudantes matriculados que também são notificados a cada alteração realizada na agenda.

A AutoReg ainda disponibiliza a Agenda do Aluno que permite a cada estudante criar sua agenda de estudo, considerando a agenda da disciplina. Nela, o aluno deve informar o conteúdo a ser estudado, data de início e término de cada atividade, local e horário de estudo, forma de estudo (individual/grupo). O sistema ainda notifica o aluno sobre as próximas atividades agendadas, bem como informa quais estão atrasadas. $\mathrm{O}$ aluno poderá configurar tais notificações definindo quando elas deverão ser enviadas. Cada atividade deverá estar associada a uma meta de aprendizagem, o aluno poderá modificar a sua agenda e o professor poderá sugerir melhorias através do envio de mensagens.

Outro requisito da ferramenta consiste na Definição e Avaliação de Metas de Aprendizagem. Assim, é necessário que o aluno crie metas de aprendizagem e definida a prioridade de cada uma delas. Uma meta de aprendizagem poderá ser associada a mais de uma atividade. O aluno deverá informar quanto tempo deseja se dedicar a cada meta de aprendizagem e o tempo total das atividades que compõem uma meta não pode exceder o tempo de dedicação previsto pelo aluno. O sistema notifica o aluno sobre o final do prazo para 
alcance de determinada meta e ao final do prazo questiona se aluno atingiu ou não a meta traçada.

Outro requisito da AutoReg envolve a Realização e Monitoramento de Atividades. Ao iniciar uma atividade, o aluno deverá alterar o status dela. Ao concluí-la deverá também realizar alteração no status da atividade. Na realização de cada atividade, o sistema permite que o aluno informe quais estratégias de aprendizagem está utilizando. O aluno poderá compartilhar com os demais alunos cadastrados a estratégia utilizada atrelada à meta de aprendizagem por ele definidas. Os demais alunos poderão comentar o material compartilhado.

Finalmente, Relatórios são disponibilizados ao professor e aos alunos possibilitando identificar o tempo dedicado a cada atividade, se a meta traçada foi alcançada pelo aluno, as estratégias de aprendizagem utilizadas em atividades desempenhas com êxito, as estratégias utilizadas em atividades em que o aluno não obteve um bom resultado. Cada aluno tem acesso ao seu próprio relatório de desempenho. O professor, no entanto, tem acesso aos relatórios de todos os alunos de sua disciplina. Os relatórios produzidos exibem informações históricas de todas as atividades realizadas pelo aluno. Ainda, possibilita a visualização do desempenho na atividade atual.

\subsection{Arquitetura}

A Figura 1 exibe a arquitetura da AutoReg composta pelos módulos Professor, Aluno e Acompanhamento/Feedback.

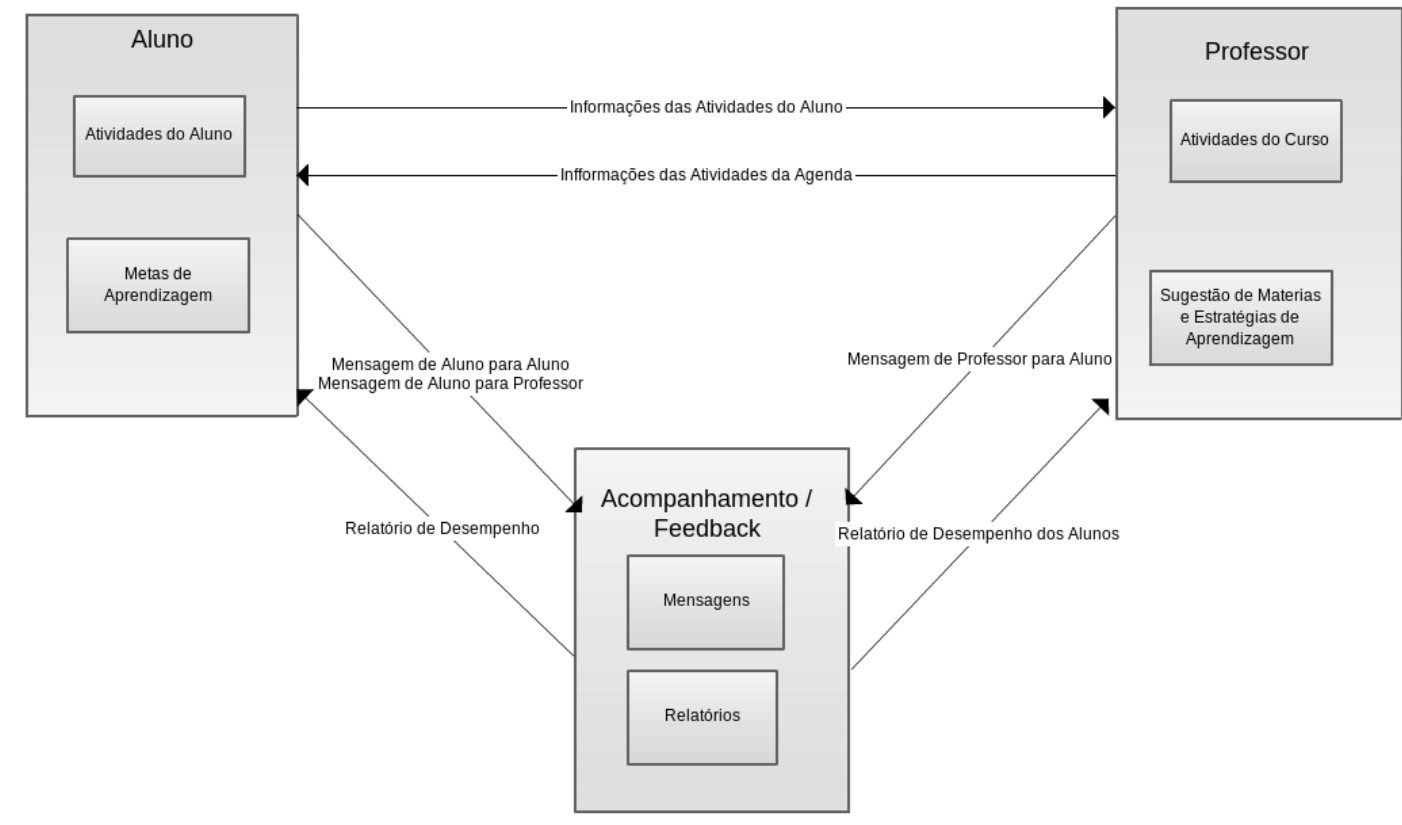

Figura 1. Arquitetura da AutoReg

Módulo Professor: é responsável pela criação da agenda da disciplina, criação e detalhamento das atividades da agenda do curso, como sugestões de materiais e estratégias de aprendizado. Este módulo, além de registrar alunos para o curso, permite uma série de interações com os estudantes, como ver a agenda pessoal de atividades, ver o relatório de desempenho e enviar mensagens para esses aprendizes.

Módulo Aluno: é responsável por fornecer informações ao estudante sobre a agenda da disciplina, como informações de cada atividade proposta pelo professor. O módulo fornece 
funcionalidades para envio de mensagens para o professor e criação da agenda pessoal do aluno, onde ele poderá associar metas de aprendizagem para cada atividade. Caso compartilhe a atividade com a turma, o aluno pode receber mensagens de outros alunos sobre aquela atividade. $\mathrm{O}$ módulo também fornece um relatório de desempenho para o aluno.

Módulo Acompanhamento/Feedback: é responsável pelo gerenciamento das mensagens entre os usuários, geração dos relatórios de desempenho e notificação aos alunos sobre alterações na agenda e atividades que chegaram ao fim do prazo.

\section{Cenário de uso}

Nesta seção serão exibidas algumas funcionalidades da AutoReg por meio de captura de telas do sistema durante sua execução. Inicialmente, considere-se que o curso Introdução à Computação já foi cadastrado na ferramenta, que João seja o professor responsável pelo curso e que José seja um dos alunos matriculados.

João criou uma atividade do curso denominada "Estruturas de Controle". Ciente disto, José deverá definir metas e atividades que o ajudem a atingir os objetivos definidos pelo professor na disciplina que, neste caso, é a compreensão de "Estruturas de Controle". Desse modo, José traçou como uma de suas metas, compreender "Expressões Condicionais", uma das habilidades necessárias para atingir o objetivo do curso. Associada a esta meta, o estudante criou atividades que serão seguidas, considerando datas de início e término, na tentativa de atingir o objetivo almejado. Uma das atividades planejadas é estudar "if - else", conforme apresentado na Figura 2.

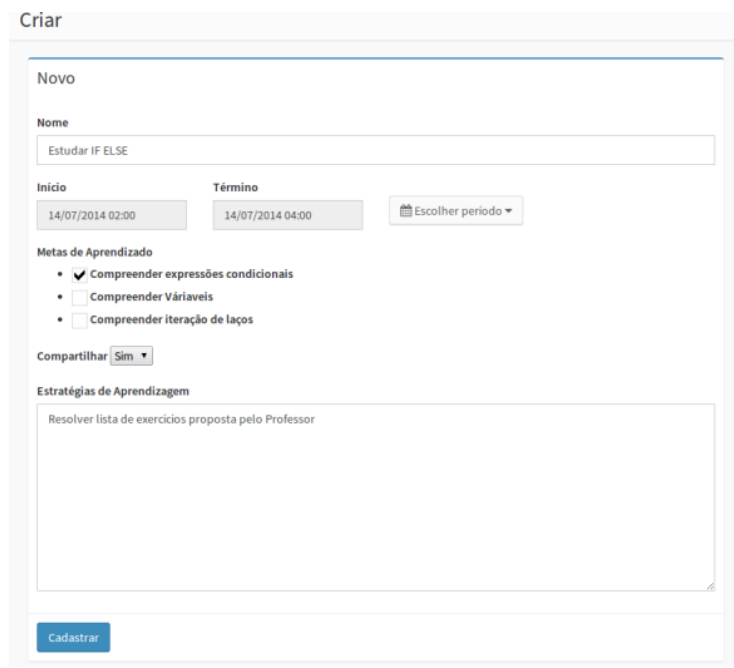

Figura 2. Criação de atividade do aluno

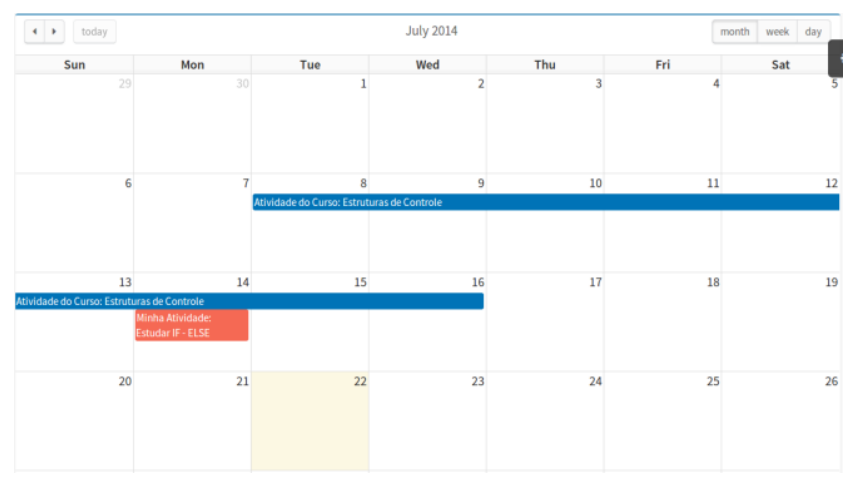

Figura 3. Agenda do aluno após criação de atividade

Como mostrado na Figura 2, para a criação da atividade estudar "if - else" o aluno deve informar o nome da atividade; datas de início e término; se deseja compartilhar a atividade com a turma para que outros alunos possam fazer comentários; e as estratégias de aprendizagem que adotará na execução da atividade. Pode-se identificar na imagem, também, metas de aprendizado que foram definidas em outro momento pelo próprio aluno. Após a criação da atividade, esta será integrada à agenda de José, a qual conterá tanto suas atividades cadastradas, destacadas em vermelho, quanto àquelas definidas pelo professor para serem cumpridas durante o curso, destacadas em azul (Figura 3).

Agora que a atividade está criada, o professor conseguirá visualizar a agenda de José e poderá enviar mensagens ao aluno sugerindo melhorias. Caso José tenha optado por 
compartilhar a sua meta de estudo e estratégias que planeja adotar para alcançá-la, o plano será exibido na página dos demais estudantes da turma que poderão também contribuir sugerindo melhorias nesse planejamento. Ao final do prazo da atividade, o qual foi definido pelo aluno, José será notificado e questionado se sua meta foi de fato atingida. A Figura 4 mostra esta notificação. É nesta fase que José avalia o próprio conhecimento e expressa se o objetivo traçado foi alcançado dentro do prazo por ele definido.

Tanto João quanto José poderão acessar o relatório de desempenho do aluno. A Figura 5 mostra a tela de relatório após o aluno informar se atingiu ou não a meta da atividade compreender "if - else".

I Final do prazo de Atividade
O prazo das seguintes atividades expiraram:
\begin{tabular}{|l|r|r|}
\hline Atividade & Meta Atingida \\
\hline Estudar IF - ELSE & Sim & Não \\
\hline
\end{tabular}

Figura 4. Notificação sobre sucesso da meta ao fim prazo

\section{Relatório}

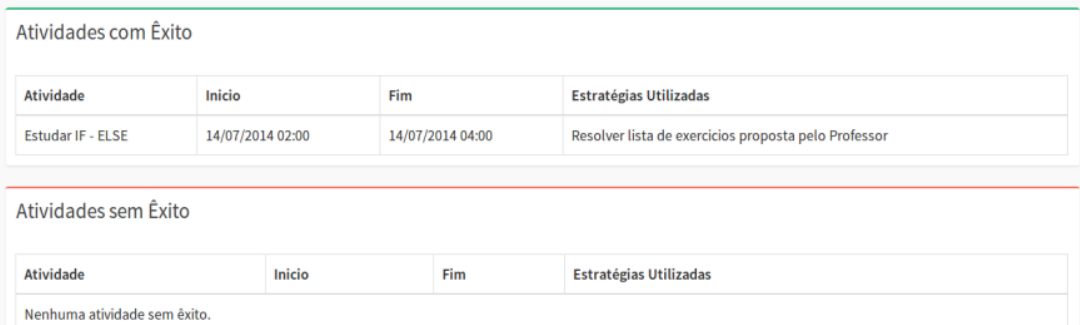

Figura 5. Relatório de acompanhamento do aluno

\section{Considerações Finais}

Este trabalho teve como objetivo apresentar a ferramenta AutoReg, a qual fornece apoio ao desenvolvimento da autorregulação da aprendizagem dos estudantes. Tal suporte é feito com as funcionalidades de gerenciamento de atividades, que contempla cadastro de disciplina, criação de agendas, definição de planos de estudos, monitoramento e avaliação do conhecimento. Também, pelo canal de comunicação implementado que possibilita a troca de mensagens entre professor e alunos, favorecendo a aprendizagem dos envolvidos.

Como trabalhos futuros pretende-se validar a ferramenta implementada, verificando o seu impacto do desenvolvimento da autorregulação dos estudantes em diferentes cenários de aprendizagem. Também, almeja-se implementar mecanismos que deem suporte à realização das atividades, de acordo com as estratégias de aprendizagem pretendidas pelos estudantes.

\section{Referências}

Alhazbi, S., Mahmood H. (2010). "Fostering Self-Regulated learning in Introductory Computer Programming Course". Disponível em $<$ http://wccce2013.ca/wpcontent/uploads/2013/05/wccce2013_submission_7.pdf>. Acesso em 22 de jul. de 2014.

Kumar, V., Winne, P., Hadwin, A., Nesbit, J., Jamieson-Noel, D., Calvert, T., \& Samin, B. (2005). "Effects of self-regulated learning in programming". In IEEE International Conference on Advanced Learning Technologies.

Lima, A. F. O. \& Pimentel, E. P. (2013). "Mecanismos para Suporte à Auto-Regulação da Aprendizagem do Estudante". In Anais do XXIV Simpósio Brasileiro de Informática e Educação.

Sirotheau, S., de Brito, S. R., da Silva, A. D. S., Eliasquevici, M. K., Favero, E. L., \& Tavares, O. D. L. (2011). "Aprendizagem de iniciantes em algoritmos e programação: foco nas competências de autoavaliação". In Anais do XXII Simpósio Brasileiro de Informática na Educação.

Zimmerman, B. J. (1990). "Self-regulated learning and academic achievement: An overview". Educational psychologist, 25(1), 3-17. 